KIAS-P98002, hep-th/9802026

\title{
The Background Geometry of DLCQ Supergravity
}

\author{
Seungjoon Hyun* \\ Department of Physics and Research Institute for Basic Sciences \\ Kyunghee University, Seoul 130-701, Korea \\ and \\ School of Physics, KIAS, Seoul 130-012, Korea
}

(February, 1998)

\begin{abstract}
By following Seiberg's prescriptions on DLCQ of $M$ theory, we give the background geometries of DLCQ supergravity associated with $N$ sector of DLCQ of $M$ theory on $T^{p}$ with vanishingly small radii. Most of these are the product of anti-de Sitter spacetimes and spheres, which have been found as the spontaneous compactifications of eleven dimensional supergravity long time ago and also are revisited recently by Maldacena by considering the near horizon geometry of various D-branes in appropriate limit. Those geometries are maximally symmetric and have full 32 supersymmetries of eleven dimensional supergravity, which agrees with the number of supersymmetries of DLCQ of $M$ theory. This suggests that DLCQ of $M$ theory is the $M /$ string theory on these nontrivial background.
\end{abstract}

*email: sjhyun@nms.kyunghee.ac.kr 


\section{INTRODUCTION}

One of the exciting developments for the formulation of eleven dimensional $M$ theory has started with the $M$ (atrix) model given in [1]. It is the formulation of $M$ theory in infinite momentum frame (IMF). The basic idea is that, in this infinitely boosted frame, the only relevant degrees of freedom are those of $N$ D0 branes and thus the effective action is given by those of $N$ D0 branes which is the dimensional reduction of ten dimensional $U(N)$ super Yang-Mills theory down to $(0+1)$-dimensions. Later Susskind has noted [2] that, instead, one can consider the $N$-sector of light-cone compactification, so-called, the discrete lightcone quantization (DLCQ) of $M$ theory which would be identical to the IMF matrix model in the large $N$ limit. Recently, the clear statement on this matrix model conjecture of $M$ theory was given by Seiberg [3] and Sen [4]. See also [5,6] for nice reviews on $M$ (atrix) theory and [7,8]. Following them, the finite $N$ DLCQ matrix model, which is compactified on light-like circle with radius $R$ and Planck scale $M_{P}$, is equivalent to $\tilde{M}$ theory with Planck scale $\tilde{M}_{P}$ compactified on a spatial circle of radius $\tilde{R}$ in the limit $\tilde{R} \rightarrow 0$, in which it becomes, naturally, the theory of $D 0$ branes.

Recently, asymptotically anti-de Sitter $(A d S)$ black hole solutions of superstring theory have got much attention. Historically, they have been realized as the solutions of supergravity via the spontaneous compactification over $S^{n}$. In their new perspectives, first noted in [9], they are realized as the solutions of type II superstring theories and are connected with the usual asymptotically flat black hole solutions by the $U$-duality transformations. See also [10,11]. Since it involves, asymptotically, light-cone compactification (i.e. periodic in light-cone coordinate), it would be natural to consider the solutions as those of DLCQ supergravity. Indeed, this kind of non-flat background geometries is the natural one to appear as one considers the supergravity limit of DLCQ of $M$ theory [12,13]. By following [3], it has been shown explicitly in [13] that if one takes DLCQ on the background five dimensional black holes with three charges, one gets effectively two-dimensional black hole solutions .

In this paper we describe the background spacetimes of the DLCQ supergravity, corre- 
sponding to the DLCQ of $M$ theory on $T^{p}$ with vanishingly small radius. We follow closely the prescriptions given in [3] and [4] and realize a wide class of the product of anti-de Sitter spacetimes and spheres as background geometries of DLCQ supergravity. Some of these are those found long time ago as the solution of spontaneous compactifications of eleven dimensional supergravity. In his recent paper [14], Maldacena shows that the same kind of various AdS spacetimes of supergravity emerges from the large $N$ limit of certain conformal field theories. Based on this, supplemented with other arguments, he conjectured that compactifications of $\mathrm{M} /$ string theory on various anti-de Sitter spaces are dual to various conformal field theories. We get these solutions as the background geometry of supergravity of $N$ sector of DLCQ $M$ theory.

In section two, we summarize some basic prescription of DLCQ of $M$ theory given in [3,4]. In section three, we consider the background geometries of various compactifications of DLCQ $M$ theory over $T^{p}$. We get the background spacetimes which are asymptotically $A d S_{4} \times S^{7}, A d S_{5} \times S^{5}, A d S_{7} \times S^{4}$ and $R^{1,6} \times S^{3}$ for $p=2,3,4,5$, respectively. In section four, we draw some conclusions.

\section{BRIEF REVIEWS ON DLCQ OF $M$ THEORY}

By following [3], we consider the compactification of $M$ theory on a light-like circle as a limit of a compactification on a small spatial circle of radius $R_{s}$, boosted by a large amount. As it has light-like compactification with the radius $R$, we can consider $x^{+}$as a genuine time coordinate. After the compactification along $x^{-}$, it becomes the ten-dimensional theory with hidden eleventh dimension with radius $R$. The prescription given in [3] and [4] is that this DLCQ of $M$ theory is equivalent to another $M$ theory, refered to as $\tilde{M}$ theory with different Planck scale $\tilde{M}_{P}$ compactified on a small spatial circle of radius $\tilde{R}$ with the identifications,

$$
M_{P} R_{i}=\tilde{M}_{P} \tilde{R}_{i}
$$

for the nine dimensional transverse space part $x^{i}$ and 


$$
\tilde{R} \tilde{M}_{P}^{2}=R M_{P}^{2}
$$

These are finite in the limit $\tilde{R} \rightarrow 0$. In this limit we have the string theory with string coupling and string scale

$$
\begin{gathered}
\tilde{g}_{s}=\left(\tilde{R} \tilde{M}_{P}\right)^{\frac{3}{2}} \sim \tilde{R}^{\frac{3}{4}} \rightarrow 0 \\
\tilde{M}_{s}^{2}=\tilde{R} \tilde{M}_{P}^{3} \sim \tilde{R}^{-\frac{1}{2}} \rightarrow \infty
\end{gathered}
$$

which becomes the theory of $N$ D0-branes, living in a small transverse space of characteristic size $\tilde{R}_{i} \sim \tilde{R}^{\frac{1}{2}} \rightarrow 0$.

For a background with compactification on $T^{p}$ one uses $\mathrm{T}$ duality so that it becomes the theory of $N \mathrm{D} p$-branes on a torus with radii

$$
\tilde{\Sigma}_{i}=\frac{1}{\tilde{R}_{i} \tilde{M}_{s}^{2}}=\frac{1}{R_{i} R M_{P}^{3}},
$$

which are finite under the limit $\tilde{R} \rightarrow 0$. The string coupling after this $\mathrm{T}$ duality transformation becomes

$$
\tilde{g}_{s}^{\prime}=\tilde{M}_{s}^{p-3}\left(R M_{P}^{2}\right)^{3} \prod \Sigma_{i} \sim \tilde{R}^{-(p-3) / 4} .
$$

The low energy effective theory for $p \leq 3$ is described by $(p+1)$-dimensional SYM with gauge coupling

$$
g_{Y M}^{2}=\frac{\tilde{g}_{s}^{\prime}}{\tilde{M}_{s}^{p-3}}=\left(R M_{P}^{2}\right)^{3} \prod \Sigma_{i}
$$

which is finite under the limit $\tilde{R} \rightarrow 0$. In the following section we use these to find the background geometry of DLCQ supergravity for $M$ theory on $T^{p}$. As it turns out, it is not the Minkowski spacetime, yet it has the full supersymmetry of eleven dimensional supergravity.

\section{DLCQ OF M THEORY ON $T^{P}$}

We consider the metric of D0 branes on $T^{p}$ in the infinite momentum frame. It is the straightforward generalization of D0 branes on noncompact space given in [13]. The D0 brane configurations of the type IIA superstring theory on $T^{p}$ is given by [15] 


$$
\begin{aligned}
d s^{2} & =-\frac{1}{\sqrt{f_{0}}} d t^{2}+\sqrt{f_{0}}\left(d x_{1}^{2}+\cdots+d x_{p}^{2}\right)+\sqrt{f_{0}}\left(d x_{p+1}^{2}+\cdots+d x_{9}^{2}\right), \\
e^{-2 \phi} & =g_{s}^{-2} f_{0}^{(p-3) / 2}
\end{aligned}
$$

where $g_{s} \equiv e^{\phi_{\infty}}$ is the string coupling constant, $x^{i}, i=1 \cdots p$, are internal $T^{p}$ coordinates with radii $R_{i}$ and a $(9-p)$-dimensional harmonic function

$$
f_{0}=1+\left(\frac{r_{0}}{r}\right)^{7-p}
$$

with the charge $r_{0}^{7-p}=\frac{Q l_{p}^{9}}{R_{s}^{2} R_{1} \cdots R_{p}}$ and $r^{2}=x_{p+1}^{2}+\cdots+x_{9}^{2}$. The dimensionless number $Q$ is proportional to $N$, the number of D-particles.

It has been shown in [13] that the field configurations in DLCQ is given by

$$
\begin{aligned}
d s^{2} & =-\frac{1}{\sqrt{f}} d t^{2}+\sqrt{f}\left(d x_{1}^{2}+\cdots+d x_{p}^{2}\right)+\sqrt{f}\left(d x_{p+1}^{2}+\cdots+d x_{9}^{2}\right), \\
e^{-2 \phi} & =g_{s}^{-2} f^{(p-3) / 2}
\end{aligned}
$$

with the new harmonic function $f$ given by

$$
f=\frac{Q l_{p}^{9}}{R^{2} R_{1} \cdots R_{p} r^{7-p}},
$$

where $R$ is the compactification radius over the light-cone circle $x^{-}$. In the generic cases, when the locations of D-particles are arbitrary, we get (8) with

$$
f=\sum_{a} \frac{Q_{a} l_{p}^{9}}{R^{2} R_{1} \cdots R_{p}\left|\vec{r}-\overrightarrow{x_{a}}\right|^{7-p}}
$$

where $Q=\sum_{a} Q_{a}$ and $r=0$ is the center of mass of D-particles.

Since we are taking the light-cone coordinate $x^{-}$as eleventh compactified direction, the string coupling and the string scale are given by

$$
\begin{gathered}
g_{s}=\left(R M_{P}\right)^{\frac{3}{2}} \\
M_{s}^{2}=R M_{P}^{3} .
\end{gathered}
$$

The prescriptions on the DLCQ of M theory given in the last section suggest that this background geometry is connected to the background geometry of $\tilde{M}$ theory with the following identifications for the metric, 


$$
\frac{1}{\tilde{\alpha}^{\prime}} d \tilde{s}^{2}=\frac{1}{\alpha^{\prime}} d s^{2}
$$

and the string coupling,

$$
\frac{\tilde{g}_{s}}{g_{s}}=\left(\frac{\tilde{\alpha}^{\prime}}{\alpha^{\prime}}\right)^{\frac{3}{2}}
$$

In terms of the coordinates of $\tilde{M}$ theory, $\tilde{x}^{m}$, (8) with the harmonic function (9) become

$$
\begin{aligned}
d \tilde{s}^{2} & =-\frac{1}{\sqrt{\tilde{f}}} d t^{2}+\sqrt{\tilde{f}}\left(d \tilde{x}_{1}^{2}+\cdots+d \tilde{x}_{p}^{2}\right)+\sqrt{\tilde{f}}\left(d \tilde{x}_{p+1}^{2}+\cdots+d \tilde{x}_{9}^{2}\right), \\
e^{-2 \tilde{\phi}} & =\tilde{g}_{s}^{-2} \tilde{f}^{-3 / 2}
\end{aligned}
$$

where

$$
\tilde{f}=\frac{Q \tilde{l}_{p}^{9}}{\tilde{R}^{2} \tilde{R}_{1} \cdots \tilde{R}_{p} \tilde{r}^{7-p}}=\left(\frac{\tilde{M}_{P}}{M_{P}}\right)^{2} f,
$$

and $\tilde{R}_{i}=\frac{M_{P}}{\tilde{M}_{P}} R_{i}$ are radii of $\tilde{x}^{i}$. This, or more general cases with

$$
\tilde{f}=\sum_{a} \frac{Q_{a} \tilde{l}_{p}^{9}}{\tilde{R}^{2} \tilde{R}_{1} \cdots \tilde{R}_{p}\left|\tilde{\vec{r}}^{2}-\tilde{\vec{x}}_{a}\right|^{7-p}},
$$

is indeed the supergravity solutions of the $\tilde{M}$ theory with the same configurations in the limit (3), which implies the validity of the prescription described in section 2 when applied to the background geometry of supergravity associated with the DLCQ of $M$ theory. This, in turn, tells us that in the DLCQ of $M$ theory we are considering the $N$-sector of $D 0$ branes whose background geometry is those given in (8) with the harmonic function $f$ given by (9) or (10). Note that in order to deal with the light-cone quantization of field theories in the flat Minkowski spacetime, we need to introduce fictitious cutoff parameter to turn the light-like coordinate into the space-like one [7,8]. In this case, on the contrary, $x^{-}$can be considered as true eleventh coordinate with the compactification radius $R$ as background D0-particles provide well-defined metric in which $x^{-}$play the role as space-like coordinate. In the followings we perform T-dualities along $T^{p}$, in the $\tilde{M}$ theory framework, and map to original DLCQ $M$ theory to get the background geometries associated with the supergravity solutions of DLCQ of $M$ theory on $T^{p}$. This turns out to be equivalent to taking T-dualities directly on the solution (8). 


\section{A. DLCQ of $M$ theory on $T^{3}$ and $A d S_{5} \times S^{5}$}

Let us first consider the $N$ sector of DLCQ M theory on $T^{3}$. As described in section 2, after performing T-dualities along $\tilde{x}^{i}$ directions on the metric (14) of $\tilde{M}$ theory, which turns the configurations into those of D3 branes, the background geometry, with the harmonic function (15) becomes

$$
d \tilde{s}^{2}=\tilde{\alpha}^{\prime}\left[\frac{U^{2}}{\sqrt{Q \tilde{g}_{s}^{\prime}}}\left(-d \tilde{t}^{2}+d \tilde{x}_{1}^{2}+d \tilde{x}_{2}^{2}+d \tilde{x}_{3}^{2}\right)+\sqrt{Q \tilde{g}_{s}^{\prime}}\left(\frac{d U^{2}}{U^{2}}+d \Omega_{5}^{2}\right)\right],
$$

where $U$ is the radial coordinate, which is well-defined in the limit $\tilde{R} \rightarrow 0$

$$
U \equiv \frac{\tilde{r}}{\tilde{\alpha}^{\prime}}=\left(R M_{P}^{3}\right) r
$$

By transforming back to the original DLCQ of $M$ theory using (12) and

$$
\tilde{g}_{s}^{\prime}=g_{s}^{\prime}=\left(R M_{P}^{2}\right)^{3} \prod_{i}^{3} \tilde{\Sigma}_{i}
$$

we end up with the configurations

$$
d s^{2}=\frac{r^{2}}{\sqrt{Q g_{s}^{\prime}} \alpha^{\prime}}\left(-d t^{2}+d x_{1}^{2}+d x_{2}^{2}+d x_{3}^{2}\right)+\sqrt{Q g_{s}^{\prime}} \alpha^{\prime}\left(\frac{d r^{2}}{r^{2}}+d \Omega_{5}^{2}\right) .
$$

As mentioned earlier, this can be derived by performing T-dualities directly on the configurations (8). Generically we use (10) and get the family of background geometries, which are asymptotically $A d S_{5} \times S^{5}$, with the metric

$$
d s^{2}=\frac{1}{\sqrt{f}}\left(-d t^{2}+d x_{1}^{2}+d x_{2}^{2}+d x_{3}^{2}\right)+\sqrt{f}\left(d x_{4}^{2}+\cdots+d x_{9}^{2}\right)
$$

where

$$
f=\sum_{a} \frac{Q_{a} g_{s}^{\prime} \alpha^{2}}{\left|\vec{r}-\overrightarrow{x_{a}}\right|^{4}}
$$

and $\vec{x}_{a}$ are moduli of DLCQ of $M$ theory on $T^{3}$. Hence the $N$-sector of DLCQ $M$ theory on $T^{3}$, which is described by the fluctuations on the $N$ D3 brane background and thus $\mathrm{N}=4$ fourdimensional Yang-Mills theory, would be related to the type IIB supergravity/superstring on the asymptotically $A d S_{5} \times S^{5}$ background (20). The radii for $x^{i}, i=1,2,3$ are given by

$$
\Sigma_{i}=\frac{\alpha^{\prime}}{R_{i}}
$$


and thus $x^{i}$ become noncompact coordinates if the original tori of DLCQ $M$ theory shrink to zero size. Then the background geometry is given by asymptotically $A d S_{5} \times S^{5}$, with no identification in spatial coordinates of $A d S$ space. In this case, the number of geometrical Killing spinors becomes 32 [16,17] thus may be treated as true vacua of the DLCQ of $M$ theory.

\section{B. DLCQ of $M$ theory on $T^{4}$ and $A d S_{7} \times S^{4}$}

Let us consider another example: $N$ sector of DLCQ $M$ theory on $T^{4}$. In this case, as can be seen from (5) with $p=4$, the string coupling divergies, therefore we need to go the strong coupling limit of $\tilde{M}$ theory, which is an eleven dimensional theory. D4 branes become M5 branes wrapping the eleventh dimension whose radius is now finite and is given by

$$
\tilde{\Sigma}_{11}=\frac{\tilde{g}_{s}^{\prime}}{\tilde{M}_{s}}=\left(R M_{P}^{2}\right)^{3} \prod \tilde{\Sigma}_{i}
$$

Therefore $N$ sector of DLCQ $M$ theory on $T^{4}$ becomes the theory of $N$ M5 branes in eleven dimensions 19 28]. The eleven dimensional Planck scale of $\tilde{M}$ theory becomes

$$
\tilde{M}_{P}^{\prime}=\frac{\tilde{M}_{s}}{\left(\tilde{g}_{s}^{\prime}\right)^{\frac{1}{3}}}=\left(\tilde{R}^{2} \tilde{R}_{1} \cdots \tilde{R}_{4} \tilde{M}_{P}^{9}\right)^{\frac{1}{3}} \sim \tilde{R}^{-\frac{1}{6}} \longrightarrow \infty
$$

and transverse radial coordinate $\tilde{r}$ scales by (1), i.e. $\tilde{r} \sim \tilde{R}^{\frac{1}{2}}$, thus well-defined radial coordinate in the limit, $\tilde{R} \rightarrow 0$, can be written as

$$
U^{2} \equiv \tilde{r}\left(\tilde{M}_{P}^{\prime}\right)^{3}=r\left(M_{P}^{\prime}\right)^{3}
$$

After taking T-dualities in $\tilde{M}$ theory, we get the background geometries of the system of D4 branes

$$
\begin{aligned}
d \tilde{s}^{2} & =\frac{1}{\sqrt{\tilde{f}}}\left(-d t^{2}+d x_{1}^{2}+\cdots+d x_{4}^{2}\right)+\sqrt{\tilde{f}}\left(d \tilde{x}_{5}^{2}+\cdots+d \tilde{x}_{9}^{2}\right), \\
e^{-2 \tilde{\phi}} & =\tilde{g}_{s}^{-2} \tilde{f}^{1 / 2}
\end{aligned}
$$

\footnotetext{
${ }^{1}$ For compact spatial coordinates, only half of supersymmetries survive [18].
} 
where

$$
\tilde{f}=\frac{Q}{\left(\tilde{M}_{P}^{\prime} \tilde{r}\right)^{3}}=Q\left(\frac{\tilde{M}_{P}^{\prime}}{U}\right)^{6} .
$$

As mentioned earlier, the eleventh dimension has finite radius (it is very large relative to the Planck scale $\left.\Sigma_{11} \tilde{M}_{P}^{\prime} \sim \tilde{R}^{-\frac{1}{6}} \rightarrow \infty\right)$, therefore it is more appropriate to consider the full eleven dimensional background geometry of $\tilde{M}$ theory. By combining boosting and rescaling back

$$
\left(M_{P}^{\prime}\right)^{2} d s^{2}=\left(\tilde{M}_{P}^{\prime}\right)^{2} d \tilde{s}^{2},
$$

we end up with the background geometry of the DLCQ of $M$ theory on $T^{4}$ which is given by

$$
d s_{11}^{2}=\left(M_{P}^{\prime}\right)^{-2}\left[\frac{U^{2}}{Q^{1 / 3}}\left(-d t^{2}+d x_{1}^{2}+\cdots+d x_{4}^{2}+d x_{11}^{2}\right)+Q^{\frac{2}{3}}\left(\frac{4 d U^{2}}{U^{2}}+d \Omega_{4}^{2}\right)\right] .
$$

Again, in the case when the moduli $\vec{x}_{a}$ are non-vanishing, we use (10) and get the background geometries which reduce to (26) in the asymptotic region $r \rightarrow \infty$. As before if one starts with vanishingly small radii of $T^{4}$ tori, the dual tori as well as the eleventh dimension become non-compact and the metric describes $A d S_{7} \times S^{4}$. Therefore $N$-sector of DLCQ of $M$ theory on vanishingly small $T^{4}$ describes supergravity/M theory on asymptotically $A d S_{7} \times S^{4}$.

\section{DLCQ of $M$ theory on $T^{2}$ and $A d S_{4} \times S^{7}$}

One can similarly work out for $T^{2}$ compactifications of DLCQ $M$ theory. In this case, after T-dualities on $T^{2}$, from (5), the eleventh dimension in $\tilde{M}$ theory has vanishingly small radius

$$
\Sigma_{11}=\left(\tilde{R}_{1} \tilde{R}_{2} \tilde{M}_{P}^{3}\right)^{-1} \sim \tilde{R}^{\frac{1}{2}}
$$

At first sight, this seems to imply that it is the theory of $N$ D2 branes in ten dimensions. However one should note that the scale of eleventh dimension, (27), is the same order of the characteristic length scale of transverse dimensions (四), which implies underlying $S O(8)$ 
structure of the theory. By going back to the DLCQ of $M$-theory on $T^{2}$ with vanishingly small radii $R_{i} \rightarrow 0$, one can easily see that the radius of eleventh dimension as well as the radii of dual tori $\hat{T}^{2}$ become non-compact, indicating the geometry is the eleven dimensional one. In this eleven dimensional geometry, the eleventh direction becomes the transverse one and therefore the seven-dimensional harmonic function becomes the eight-dimensional one and thus can be written as

$$
f=\frac{Q l_{p}^{9}}{R^{2} R_{1} R_{2} r^{5}}=\frac{Q l_{p}^{\prime 6}}{\Sigma_{11} r^{5}} \longrightarrow \frac{Q l_{p}^{\prime 6}}{r^{6}} .
$$

The background metric of DLCQ supergravity for the system of $N$ M2 branes becomes those of $A d S_{4} \times S^{7}$,

$$
d s_{11}^{2}=\left(M_{P}^{\prime}\right)^{-2}\left[\frac{U^{2}}{Q^{2 / 3}}\left(-d t^{2}+d x_{1}^{2}+d x_{2}^{2}\right)+Q^{\frac{1}{3}}\left(\frac{d U^{2}}{4 U^{2}}+d \Omega_{7}^{2}\right)\right]
$$

where $U=r^{2}\left(M_{P}^{\prime}\right)^{3}$. As before, if we start with (10) considering the cases nonvanishing $\vec{x}_{a}$, we end up with the background geometries which are asymptotically $A d S_{4} \times S^{7}$.

\section{DLCQ of $M$ theory on $T^{5}$ and $R^{1,6} \times S^{3}$}

Finally we consider DLCQ $M$ theory on $T^{5}$ [22,29]. In the $\tilde{M}$ theory, after T-duality along $T^{5}$, one can see from (5) that it becomes the strong coupling limit of D5 branes in type IIB theory. Therefore we need to perform the S-duality and then it becomes the theory of $N$ NS5 branes in type IIB theory with vanishingly small string coupling,

$$
\bar{g}_{s}=\left(\tilde{g}_{s}^{\prime}\right)^{-1} \sim \tilde{R}^{\frac{1}{2}}
$$

and finite string scale,

$$
M_{s}^{2}=\frac{\tilde{M}_{s}^{2}}{\tilde{g}_{s}^{\prime}}=R^{2}\left(R_{1} \cdots R_{5}\right) M_{P}^{9} .
$$

The appropriate background geometry can be found accordingly, by using (12), and in the limit, $\operatorname{vol}\left(T^{5}\right) \rightarrow 0$ of original $T^{5}$ torus, it is given by the product of Minkowskian spacetimes $R^{1,6}$ and $S^{3}$, 


$$
d s_{10}^{2}=-d t^{2}+d x_{1}^{2}+\cdots+d x_{5}^{2}+\frac{Q}{M_{s}^{2}} d \rho^{2}+\frac{Q}{M_{s}^{2}} d \Omega_{3}^{2}
$$

where the well-defined radial coordinate $U=\frac{\tilde{r}}{\bar{g}_{s}}=\frac{r}{g_{s}}$ is replaced by $\rho=\log \left(M_{s} U\right)$. As shown in [13], if we have additional branes, the geometry describes effective two-dimensional CGHS type black holes [30]. For example, consider the same compactification with extra D4 branes. Along the same line of arguments with S-duality and T-dualities on the transverse directions, we end up with the system of NS5 branes with extra fundamental strings, whose geometry is given by the metric of the form,

$$
d s_{10}^{2}=\frac{1}{f_{1}}\left(-d t^{2}+d x_{1}^{2}\right)+d x_{2}^{2}+\cdots+d x_{5}^{2}+Q \alpha^{\prime} \frac{d U^{2}}{U^{2}}+Q \alpha^{\prime} d \Omega_{3}^{2},
$$

where

$$
f_{1}=1+\frac{Q_{4} \Sigma_{5}\left(R M_{P}^{2}\right)^{3} \alpha^{\prime 2}}{U^{2}}
$$

Here $Q_{4}$ is proportional to the number of D4 branes which turns into fundamental strings after the chain of S- and T-dualities.

\section{CONCLUSIONS}

In this paper we have described the background geometry of DLCQ supergravity associated with DLCQ $M$ theory. In contrast to the usual situation, like in ordinary string theory, in which we consider the flat Minkowskian spacetime as a true vacuum with full supersymmetry and consider various excitations on those backgrounds, the N sector of DLCQ $M$ theory should be interpreted as having N D0 branes (for non-compact spacetimes and Dp-branes on $T^{p}$ ) as background with full supersymmetries. Therefore various spacetimes found above should be considered as the background geometries on which the theory should be defined. Our results remain true for any $N$, though we generally need $N$ to be large in order to be in supergravity regime. In the large $N$ limit, $M$ (atrix) theory conjecture tells us that it is equivalent to the $M$ theory with non-compact eleventh dimension. Our results, which also holds in the large $N$ limit, suggest that the $N$-sector of DLCQ of $M$ theory 
and its large $N$ limit should be considered as $M /$ string theory defined on these non-trivial, typically asymptotically $(A d S)_{p} \times S^{q}$, background. The derivation of [3, []] is independent of the number of supersymmetries. Therefore all the discussions in this paper can be applied

for the non-supersymmetric cases as well. For example one may begin with D0-anti-D0 bound state in DLCQ on $T^{p}$. Then by following the above procedures, one ends up with non-extremal anti-de Sitter black hole solutions.

\section{ACKNOWLEDGMENTS}

I have benefited from comments by Jin-Ho Cho, Youngjai Kiem, Sangmin Lee and JaeSuk Park. I also would like to thank Juan Maldacena who pointed out an error in previous version. This work was supported in part by Korea Research Foundation. 


\section{REFERENCES}

[1] T. Banks, W. Fischler, S. H. Shenker, L. Susskind, Phys. Rev. D 55 (1997) 5112.

[2] L. Susskind, hep-th/9704080.

[3] N. Seiberg, Phys. Rev. Lett. 79 (1997) 3577, hep-th/9710009.

[4] A. Sen, hep-th/9709220.

[5] T. Banks, hep-th/9710231.

[6] D. Bigatti and L. Susskind, hep-th/9712072.

[7] S. Hellerman and J. Polchinski, hep-th/9711037.

[8] D. Bigatti and L. Susskind, hep-th/9711063.

[9] S. Hyun, hep-th/9704005.

[10] H.J. Boonstra, B. Peeters and K. Skenderis, Phys. Lett. 411B (1997) 59, hepth/9706192; hep-th/9801076.

[11] K. Sfetsos and K. Skenderis, hep-th/9711138.

[12] K. Becker, M. Becker, J. Polchinski and A. Tseytlin, Phys. Rev. D 56 (1997) 3174, hep-th/9711037.

[13] S. Hyun, H. Shin and Y. Kiem, Phys. Rev. D 57 (1998) 4856, hep-th/9712021.

[14] J.M. Maldacena, hep-th/9711200.

[15] G. T. Horowitz and A. Strominger, Nucl. Phys. B360 (1991) 197.

[16] R. Kallosh and J. Kumar, Phys. Rev. D 56 (1997) 4934

[17] R. Haag, J. Lopuszanski and M. Sohnius, Nucl. Phys. B88 (1975) 257

[18] H. Lü, C.N. Pope and P.K. Townsend, hep-th/9607164 
[19] E. Witten, hep-th/9507121.

[20] A. Strominger, Phys. Lett. B383 (1996) 44.

[21] M. Rozali, Phys. Lett. B400 (1997) 260, hep-th/9702136.

[22] M. Berkooz, M. Rozali and N. Seiberg, Phys. Lett. B408 (1997) 105, hep-th/9704089.

[23] O. Aharony, M. Berkooz, S. Kachru, N. Seiberg and E. Silverstein, hep-th/9707079.

[24] E. Witten, hep-th/9707093.

[25] J. Brodie and S. Ramgoolam, hep-th/9711001.

[26] P. Claus, R. Kallosh and A. Van Proeyen, hep-th/9711161.

[27] O. J. Ganor and S. Sethi, hep-th/9712071.

[28] O. Aharony, M. Berkooz and N. Seiberg, hep-th/9712117.

[29] N. Seiberg, hep-th/9705221.

[30] C.G. Callan, S. Giddings, J. Harvey, A. Strominger, Phys. Rev. D 45 (1992) 1005. 